\title{
Numerical forcing scheme to generate passive scalar mixing on the centerline of turbulent round jets in a triply periodic box
}

\author{
Kyupaeck Jeff Rah $\odot^{*}$ and Guillaume Blanquart ${ }^{\dagger}$ \\ Department of Mechanical and Civil Engineering, California Institute of Technology, California 91125, USA
}

(Received 2 May 2019; published 30 December 2019)

\begin{abstract}
Numerical studies of passive scalars in three-dimensional (3D) periodic box turbulence have often used arbitrary scalar forcing schemes to sustain the variance. These existing methods represent certain flow configurations, but they have not been derived using specific velocity and scalar profiles. In this work, a forcing technique is devised to generate centerline scalar mixing of round jets in a triply periodic box. It is derived from the scalar transport equation using a Reynolds-like decomposition of the scalar field. The equation is closed by applying the known mean velocity and scalar profiles of axisymmetric jets. The result is a combination of a mean gradient term and a linear scalar term. Direct numerical simulations at different $\operatorname{Re}_{\lambda}$ have been performed with these source terms for unity Schmidt numbers. Scalar flux values and scaling exponents of energy spectra from simulations are comparable to experimental values. In addition, a dimensional analysis shows that the normalized scalar statistics, such as variance, flux, and dissipation rate, should only be a function of Reynolds number; indeed, such quantities computed from our simulations approach constant values as the Reynolds number increases. The effects of velocity forcing on scalar fields are also investigated; changing velocity forcing terms may result in unstable scalar fields even under the same scalar forcing. It may indicate that an appropriate relation between the velocity and scalar forcing schemes can help producing a proper scalar mixing environment.
\end{abstract}

DOI: 10.1103/PhysRevFluids.4.124504

\section{INTRODUCTION}

Passive scalars refer to diffusive scalar quantities that are convected by the velocity field but do not influence the fluid motion itself. The transport of passive scalars in an incompressible flow is governed by the following equation:

$$
\frac{\partial C}{\partial t}+\boldsymbol{u} \cdot \nabla C=\nabla \cdot(D \nabla C)
$$

where $C$ is the scalar, $D$ is its diffusivity, and $\boldsymbol{u}$ is the velocity. Numerical computations are often used to examine the mixing characteristics of passive scalars, but the computational cost and complexity of geometry can make it difficult to conduct them. To cope with these problems, a three-dimensional (3D) periodic box with zero mean scalar and velocity has been used intensively to study scalar turbulence [1-12]. Unfortunately, the solution of Eq. (1) with triply periodic boundary conditions is that of a decaying scalar field.

\footnotetext{
*krah@caltech.edu

†http://www.theforce.caltech.edu
} 
Scalar variance can be prevented from decaying by including a source term in the scalar transport equation. Below is the incompressible advection-diffusion equation with a forcing term:

$$
\frac{\partial C^{\prime}}{\partial t}+\boldsymbol{u} \cdot \nabla C^{\prime}=\nabla \cdot\left(D \nabla C^{\prime}\right)+f,
$$

where $C^{\prime}$ is the forced scalar and $f$ is a forcing term. Three main schemes have been proposed in the literature: mean gradient forcing (MG) [1], linear scalar forcing (LS) [11], and reaction analogy forcing (RA) [12].

The mean gradient forcing technique (MG) [1] models a passive scalar in the presence of a mean gradient $\boldsymbol{G}$ (e.g., $\langle-g, 0,0\rangle$ ) across the scalar field. The forcing term can be derived by imposing a mean gradient:

$$
C=C^{\prime}+\boldsymbol{G} \cdot \boldsymbol{x} .
$$

Then, the derived forcing term is $f=-\boldsymbol{u} \cdot \boldsymbol{G}$. Various numerical studies with the MG method have been conducted to examine the scalar mixing characteristics over a range of Schmidt numbers [1-4], with a particular emphasis on scalar flux [5], dissipation [6,7], spectrum [8], and structure functions $[9,10]$.

The linear scalar forcing technique (LS) [11] aims to capture the nature of decaying turbulence. The derivation of the forcing term begins by normalizing the scalar field:

$$
C^{\prime}=C \sqrt{\frac{\sigma_{t}^{2}}{\sigma_{c}^{2}}},
$$

where $\sigma_{t}^{2}$ is the targeted arbitrary scalar variance and $\sigma_{c}^{2}$ is the unforced scalar variance. When Eq. (4) is applied to Eq. (1) with an approximation that $\sigma_{t}^{2}=\sigma^{2}$ in the long-time limit, the forcing term $f=\frac{1}{2} \frac{\bar{\chi}}{\sigma^{2}} C^{\prime}$ is derived, where $\chi \equiv 2 D\left|\nabla C^{\prime}\right|^{2}$ is the scalar dissipation rate and $\sigma^{2}$ is the variance of $C^{\prime}$. The overline - denotes ensemble averaging.

The reaction analogy forcing technique (RA) [12] models a hypothetical chemical reaction that reverts the mixing process. The forcing term is $f=\operatorname{sign}(C) f_{c}|C|^{n}(1-|C|)^{m}$, where $m$ and $n$ are the stoichiometric coefficients of the hypothetical reaction. $f_{c}=2 m K / 2^{m}$, where $K$ is the reaction rate constant.

Although these three forcing methods successfully maintain scalar variance at a certain value, their derivations do not involve any information about velocity fields, nor is there any relation between the scalar forcing and the velocity forcing. The main objective of the current work is to introduce a scalar forcing scheme that generates the mixing of a specific practical flow. The target mixing is that of a fully developed turbulent round jet in the centerline region with unity Schmidt number.

In Sec. II, we will review the velocity forcing scheme of Rah et al. [13], which the current scalar forcing is based on. In Sec. III, the forcing term is derived, and a dimensional analysis is provided. In Sec. IV, simulation results are presented and compared against experiments. Finally, in Sec. V, the effects of velocity forcing on the scalar field are discussed.

\section{REVIEW OF JET CENTERLINE (JC) VELOCITY FORCING}

A velocity forcing scheme has been developed recently [13] to generate the turbulence of a round jet in a 3D periodic box. Since a similar derivation approach will be taken in the present work for the development of the scalar forcing terms, the derivation of the jet velocity forcing scheme is reviewed first.

The derivation begins with Lundgren's idea [14] to obtain the momentum equation for the fluctuating velocity $\boldsymbol{u}^{\prime}$ from the Navier-Stokes equation by subtracting the mean of the equation:

$$
N\left(\overline{\boldsymbol{u}}+\boldsymbol{u}^{\prime}\right)-\overline{N\left(\overline{\boldsymbol{u}}+\boldsymbol{u}^{\prime}\right)},
$$


where $N$ is the set of Navier-Stokes equations and $\overline{\boldsymbol{u}}$ is the mean velocity. Equation (5) gives

$$
\frac{\partial \boldsymbol{u}^{\prime}}{\partial t}+\overline{\boldsymbol{u}} \cdot \nabla \boldsymbol{u}^{\prime}+\boldsymbol{u}^{\prime} \cdot \nabla \overline{\boldsymbol{u}}+\boldsymbol{u}^{\prime} \cdot \nabla \boldsymbol{u}^{\prime}-\nabla \cdot \overline{\boldsymbol{u}^{\prime} \boldsymbol{u}^{\prime}}=-\frac{1}{\rho} \nabla p^{\prime}+v \nabla^{2} \boldsymbol{u}^{\prime},
$$

where $v$ is the kinematic viscosity of the fluid. Lundgren argued that it might be appropriate to use a source term of the form $\boldsymbol{f}_{u}=A \boldsymbol{u}^{\prime}$ with an arbitrary constant $A$, since the energy production term $\boldsymbol{u}^{\prime} \cdot \nabla \overline{\boldsymbol{u}}$ is linear to $\boldsymbol{u}^{\prime}$.

Rah et al. [13], on the other hand, examined all the terms in Eq. (6) by using the known mean velocity information of a round jet from $[15,16]$

$$
\begin{gathered}
\bar{u}_{x}=U_{c} \frac{F^{\prime}(\eta)}{\eta}, \\
\bar{u}_{r}=U_{c}\left[F^{\prime}(\eta)-\frac{1}{\eta} F(\eta)\right],
\end{gathered}
$$

where $U_{c}$ is the centerline mean velocity, $\eta=r / x$ is the ratio of a radial coordinate to an axial coordinate, and $F(\eta)$ is a function to be determined by the experiment.

It should be highlighted that the forcing term was developed to be used in a cubic box with triply periodic boundary conditions, in which the flow is statistically homogeneous, while in a practical round jet, the magnitudes of velocities decrease with the axial direction as $1 / x$. Thus, the following normalized Reynolds decomposition was suggested to make the fluctuating velocities suitable for periodic boundary conditions:

$$
\begin{aligned}
& u_{x}^{*}=\frac{x}{x_{o}}\left(u_{x}-\bar{u}_{x}\right) \exp \left(1-\frac{x}{x_{o}}\right) \\
& u_{i}^{*}=\frac{x}{x_{o}}\left(u_{i}-\bar{u}_{i}\right),
\end{aligned}
$$

where $x_{o}$ is the axial location, $u_{x}$ is the original longitudinal velocity, and $u_{i}$ is either of the transverse velocities of a jet. The asterisk $\left(^{*}\right)$ denotes the normalized quantity. The exponential term in the longitudinal normalization is added to enforce $\nabla \cdot \boldsymbol{u}^{*}=0$.

Equation (9) is now applied to Eq. (6), and the known mean velocity profiles of turbulent round jets [15] are also applied to $\overline{\boldsymbol{u}}$. Each term is then evaluated at $r=0$ and $x=x_{o}$. After some simplification for high Reynolds numbers, the forcing term takes the following form:

$$
\boldsymbol{f}_{u}=\frac{1}{2} \frac{U_{c}}{x_{o}}\left(2 u_{x}^{*} \hat{\mathbf{i}}+u_{y}^{*} \hat{\mathbf{j}}+u_{z}^{*} \hat{\mathbf{k}}\right)
$$

where $\hat{\mathbf{i}}, \hat{\mathbf{j}}$, and $\hat{\mathbf{k}}$ are the unit vectors in Cartesian coordinates. As apparent in the form of the source term, the simulation has a target experiment. Its $U_{c}$ and $x_{o}$ values determine the coefficients of the forcing term. This jet centerline (JC) velocity forcing term was found to successfully create turbulence in a $3 \mathrm{D}$ periodic box, whose turbulent characteristics resemble those of a round jet on the centerline.

Since the forcing coefficient $U_{c} / x_{o}$ and the computational domain length $L_{x}$ are the only inputs into these simulations, they determine the output turbulent parameters. An a priori analysis [13] provided algebraic expressions for key turbulent quantities:

$$
\begin{gathered}
k_{o}=\frac{27}{8}\left(\frac{U_{c}}{x_{o}}\right)^{2}\left(1+\frac{\left\langle\left\langle u_{x}^{* 2}\right\rangle\right\rangle}{\left\langle\left\langle\boldsymbol{u}^{* 2}\right\rangle\right\rangle}\right)^{2} l_{o}^{2}, \\
\varepsilon_{o}=\frac{27}{8}\left(\frac{U_{c}}{x_{o}}\right)^{3}\left(1+\frac{\left\langle\left\langle u_{x}^{* 2}\right\rangle\right\rangle}{\left\langle\left\langle\boldsymbol{u}^{* 2}\right\rangle\right\rangle}\right)^{3} l_{o}^{2}, \\
l_{o}=\left(\frac{2}{3} k_{o}\right)^{\frac{3}{2}} / \varepsilon_{o},
\end{gathered}
$$


where $k_{o}, \varepsilon_{o}, l_{o}$ are the expected values for the kinetic energy, dissipation rate, and integral length scale, respectively. $\langle\langle\cdot\rangle\rangle$ denotes time averaging of volume-averaged values. The ratio $\left\langle\left\langle u_{x}^{* 2}\right\rangle\right\rangle /\left\langle\left\langle\boldsymbol{u}^{* 2}\right\rangle\right\rangle$ and $l_{o}$ are outputs of the numerical simulations, which are found to be constant independently of Reynolds numbers:

$$
\begin{gathered}
l_{o} \approx 0.24 L_{x}, \\
\frac{\left\langle\left\langle u_{x}^{* 2}\right\rangle\right\rangle}{\left\langle\left\langle\boldsymbol{u}^{* 2}\right\rangle\right\rangle} \approx 0.49 .
\end{gathered}
$$

Thus, Eqs. (11) and (12) show that

$$
\begin{aligned}
& k_{o} \propto\left(\frac{U_{c}}{x_{o}}\right)^{2} L_{x}^{2}, \\
& \varepsilon_{o} \propto\left(\frac{U_{c}}{x_{o}}\right)^{3} L_{x}^{2} .
\end{aligned}
$$

The Reynolds number of the simulations can also be predicted a priori. The root mean square velocity fluctuation, $u_{\mathrm{rms}}$, the Taylor microscale, $\lambda$, and the Taylor-microscale Reynolds number, $\operatorname{Re}_{\lambda}$, are defined as

$$
\begin{gathered}
u_{\mathrm{rms}}=\sqrt{\frac{2 k}{3}}, \\
\lambda=\sqrt{15 \frac{v}{\varepsilon}} u_{\mathrm{rms}}, \\
\operatorname{Re}_{\lambda}=\frac{\lambda u_{\mathrm{rms}}}{v} .
\end{gathered}
$$

From Eq. (11), the expected value for $\operatorname{Re}_{\lambda}$ can be found to be

$$
\operatorname{Re}_{\lambda}^{o}=\sqrt{\frac{45}{2 v} \frac{U_{c}}{x_{o}}\left(1+\frac{\left\langle\left\langle u_{x}^{* 2}\right\rangle\right\rangle}{\left\langle\left\langle\boldsymbol{u}^{* 2}\right\rangle\right\rangle}\right) l_{o}^{2}} .
$$

More details on the derivations can be found in Ref. [13].

\section{PROPOSED FORCING TERM}

\section{A. Derivation of the forcing term}

In a similar fashion to the velocity case introduced in Sec. II, the goal is to derive a scalar source term that generates turbulent mixing similar to that of a round jet on the centerline. This methodology is developed to be used in a cubic box with periodic boundary conditions. The passive scalar inside the box will be homogeneous with zero mean. In a practical round jet, however, the fluctuating scalar $C^{\prime}$ is known to decrease with the axial direction as $1 / x$ [15]. Thus, to make the scalar appropriate for homogeneity and periodic boundary conditions, the following normalization is suggested:

$$
C^{*}=\frac{x}{x_{o}}(C-\bar{C})
$$

where $C$ is the original scalar and $\bar{C}$ is the mean scalar.

With Eqs. (9) and (22), we can obtain the governing equation for the scalar fluctuation from the incompressible advection-diffusion equation, Eq. (1). Then, each term can be evaluated on the centerline (i.e., $r=0$ ) and at $x=x_{o}$ by using the mean profiles for $\overline{\boldsymbol{u}}$ from Refs. $[15,16]$ and $\bar{C}$ from 
Refs. [17-21]:

$$
\bar{C}=C_{c} \exp \left[-\gamma_{1}(r / x)^{2}\right],
$$

where $C_{c}$ is the centerline mean scalar and $\gamma_{1}$ is a constant to be determined by the experiment. The result is

$$
\begin{aligned}
& \frac{\partial C^{*}}{\partial t}+\boldsymbol{u}^{*} \cdot \nabla C^{*}-\nabla \cdot\left(D \nabla C^{*}\right) \\
& \quad=\frac{U_{c}}{x_{o}} C^{*}+\frac{C_{c}}{x_{o}} u_{x}^{*}+\overline{\boldsymbol{u}^{*} \cdot \nabla C^{*}}+\frac{u_{x}^{*} C^{*}}{x_{o}}-\frac{\overline{u_{x}^{*} C^{*}}}{x_{o}}+2 D\left(\frac{C^{*}}{x_{o}^{2}}-\frac{1}{x_{o}} \frac{\partial C^{*}}{\partial x}\right) .
\end{aligned}
$$

Each term of Eq. (24) impacts the distribution of $C^{*}$ to a different extent. For the current study, it is our intent to retain only those terms significantly contributing to the first and second moments of $C^{*}$. Other terms will be considered negligible.

First, $\overline{\boldsymbol{u}^{*} \cdot \nabla C^{*}}$ and $\overline{u_{x}^{*} C^{*}} / x_{o}$ appear as $\overline{C^{*}} \overline{\boldsymbol{u}^{*} \cdot \nabla C^{*}}$ and $\overline{C^{*}} \overline{u_{x}^{*} C^{*}} / x_{o}$, respectively, in the scalar variance equation. They do not contribute to the mean scalar variance, because $\overline{C^{*}} \equiv 0$. Similarly, $u_{x}^{*} C^{*} / x_{o}$ appears as $\overline{u_{x}^{*} C^{* 2}} / x_{o}$ in the variance equation. The magnitude of $\overline{u_{x}^{*} C^{* 2}} / x_{o}$ can be compared to that of $U_{c} \overline{C^{* 2}} / x_{o}$. According to experiments [22-24], the value of $\overline{u_{x}^{\prime} C^{\prime 2}} / U_{c} C_{c}^{2}$ is about 0.0002-0.0008 and that of $\overline{C^{\prime 2}} / C_{c}^{2}$ is about $0.036-0.044$. Then, the ratio of $\overline{u_{x}^{\prime} C^{\prime 2}} / U_{c} C_{c}^{2}$ to $\overline{C^{\prime 2}} / C_{c}^{2}$ ranges from 0.0005 to 0.002 . Although Eq. (24) is for the normalized quantities, $\boldsymbol{u}^{*}$ and $C^{*}$, we will assume here that turbulent parameters of normalized quantities are comparable to those of $\boldsymbol{u}^{\prime}$ and $C^{\prime}$. Thus, we conclude that $\overline{u_{x}^{*} C^{*}} / x_{o}$ is also negligible.

Next, $D C^{*} / x_{o}^{2} \ll U_{c} C^{*} / x_{o}$ for high Reynolds number and/or high Schmidt number flows, where the Schmidt number is defined as $\mathrm{Sc} \equiv v / D$. The ratio $U_{c} x_{o} / D$ is proportional to the jet Péclet number, $\mathrm{Pe}_{D}=\operatorname{Re}_{D} \cdot \mathrm{Sc}=U_{o} d / D$, based on the exit nozzle velocity, $U_{o}$, and the nozzle diameter, $d$ [15]. Similarly, $\frac{D}{x_{o}} \frac{\partial C^{*}}{\partial x} \ll \boldsymbol{u}^{*} \cdot \nabla C^{*}$. The ratio of the two terms is also of the same magnitude as the Péclet number.

With these simplifications, the only terms significantly contributing to the scalar variance are $\frac{U_{c}}{x_{o}} C^{*}$ and $\frac{C_{c}}{x_{o}} u_{x}^{*}$. Thus, only these two terms are retained on the right-hand side of the governing equation for the passive scalar:

$$
\frac{\partial C^{*}}{\partial t}+\boldsymbol{u}^{*} \cdot \nabla C^{*}-\nabla \cdot\left(D \nabla C^{*}\right)=\frac{U_{c}}{x_{o}} C^{*}+\frac{C_{c}}{x_{o}} u_{x}^{*} .
$$

Under the assumption of statistical homogeneity, we obtain the scalar variance equation from Eq. (25):

$$
\frac{d \overline{C^{* 2}}}{d t}=-\bar{\chi}+2 \frac{U_{c}}{x_{o}} \overline{C^{* 2}}+2 \frac{C_{c}}{x_{o}} \overline{u_{x}^{*} C^{*}},
$$

where $\chi \equiv 2 D \nabla C^{*} \cdot \nabla C^{*}$ is the scalar dissipation rate. By construction, ensemble-averaged quantities should be statistically stationary in the considered region. Then, Eq. (26) becomes

$$
\bar{\chi}=2 \frac{U_{c}}{x_{o}} \overline{C^{* 2}}+2 \frac{C_{c}}{x_{o}} \overline{u_{x}^{*} C^{*}}
$$

\section{B. Properties of the forcing term}

This current forcing term, $\frac{U_{c}}{x_{o}} C^{*}+\frac{C_{c}}{x_{o}} u_{x}^{*}$, is the result of applying the physical laws of a practical turbulent flow. The mean velocity and scalar profiles of a round jet, Eqs. (7)-(23), have been used during the derivation. The target experiment determines the coefficients of the source terms with its $U_{c}, C_{c}$, and $x_{o}$ values. They control the magnitudes and characteristics of scalar turbulent quantities in a $3 \mathrm{D}$ box, which should be similar to those of a round jet on the centerline.

This forcing term is also a combination of two previously existing methods: the linear scalar forcing (LS) and mean gradient forcing (MG). $\frac{U_{c}}{x_{o}} C^{*}$ is linear to a scalar like LS, and $\frac{C_{c}}{x_{o}} u_{x}^{*}$ is linear 
TABLE I. Inputs and outputs of DNS.

\begin{tabular}{lcccc}
\hline \hline Input & $\frac{U_{c}}{x_{o}}$ & $\frac{C_{c}}{x_{o}}$ & $L_{x}$ & $v$ \\
& {$\left[T^{-1}\right]$} & {$\left[\theta L^{-1}\right]$} & {$[L]$} & {$\left[L^{2} T^{-1}\right]$} \\
Output & $\overline{C^{* 2}}$ & $\overline{u_{x}^{*} C^{*}}$ & $\bar{\chi}$ & \\
& {$\left[\theta^{2}\right]$} & {$\left[\theta L T^{-1}\right]$} & {$\left[\theta^{2} T^{-1}\right]$} & \\
\hline \hline
\end{tabular}

to a velocity like MG. Although the coefficients are arbitrary for the original MG and LS terms, they have the same forms as the current forcing term. It should be noted that this similarity is only a result of applying the physics of a practical flow.

\section{Dimensional analysis}

There are four input parameters for the simulations: the coefficients for the forcing terms, $U_{c} / x_{o}$ and $C_{c} / x_{o}$, the domain width $L_{x}$, and viscosity $v$. These four parameters control the outcomes of the simulations, such as $\overline{C^{* 2}}, \overline{u_{x}^{*} C^{*}}$, or $\bar{\chi}$. It is important to note that these three quantities are not independent and are related through the scalar variance equation, Eq. (27). Table I summarizes the inputs and outputs and their units. $T$ represents a time unit; $L$, a length unit; and $\theta$, a scalar unit.

We can apply Buckingham $\pi$ theorem to a set of four inputs and one outcome. For example, there exists a function $g$ of relating $\overline{C^{* 2}}$ to the four input parameters:

$$
g\left(\overline{C^{* 2}}, \frac{U_{c}}{x_{o}}, \frac{C_{c}}{x_{o}}, L_{x}, v\right)=0 .
$$

We suggest two nondimensional groups:

$$
\begin{gathered}
\pi_{1} \equiv \frac{\overline{C^{* 2}}}{\left(C_{c} / x_{o}\right)^{2} L_{x}^{2}}, \\
\pi_{2} \equiv \frac{\left(U_{c} / x_{o}\right) L_{x}^{2}}{v} .
\end{gathered}
$$

$\pi_{2}$ is proportional to $\operatorname{Re}_{D}$, because $L_{x}=0.399 x_{o}$ [13], and $U_{c} x_{o} / v$ is proportional to $\operatorname{Re}_{D}$ [15], as discussed in Sec. III A. Then, by the Buckingham $\pi$ theorem,

$$
\overline{C^{* 2}}=\alpha_{1}\left(\operatorname{Re}_{\lambda}\right)\left(\frac{C_{c}}{x_{o}}\right)^{2} L_{x}^{2} .
$$

$\alpha_{1}$ is a scaling coefficient, which may be a function of the Reynolds number. $\operatorname{Re}_{D}$ is replaced by $\operatorname{Re}_{\lambda}$, because it is found that $\operatorname{Re}_{\lambda} \propto \operatorname{Re}_{D}^{1 / 2}$ for turbulent round jets [25,26].

A similar procedure is used to suggest the following relation for $\overline{u_{x}^{*} C^{*}}$ :

$$
\overline{u_{x}^{*} C^{*}}=\alpha_{2}\left(\operatorname{Re}_{\lambda}\right)\left(\frac{U_{c}}{x_{o}}\right)\left(\frac{C_{c}}{x_{o}}\right) L_{x}^{2} .
$$

This suggestion comes from Eq. (31) and the fact that $\sqrt{\overline{u_{x}^{* 2}}} \propto\left(U_{c} / x_{o}\right) L_{x}$ from Eq. (11).

The final relation is for $\bar{\chi}$ :

$$
\bar{\chi}=\alpha_{3}\left(\operatorname{Re}_{\lambda}\right)\left(\frac{U_{c}}{x_{o}}\right)\left(\frac{C_{c}}{x_{o}}\right)^{2} L_{x}^{2} .
$$

This relation comes from Eqs. (27), (31), and (32). Section IV will examine the three coefficients, $\alpha_{1}, \alpha_{2}$, and $\alpha_{3}$. 
TABLE II. Relevant parameters of the target experiments and the corresponding simulations.

\begin{tabular}{|c|c|c|c|c|c|c|c|c|}
\hline & \multicolumn{4}{|c|}{ Target experiments } & \multicolumn{4}{|c|}{ Simulation parameters } \\
\hline & $U_{c} / x_{o}$ & $C_{c} / x_{o}$ & $v=D$ & $\operatorname{Re}_{D}$ & $L$ & $\kappa_{\max } \eta$ & $N$ & $\operatorname{Re}_{\lambda}$ \\
\hline DNS1 & 0.0212 & 2.41 & $1.64 \times 10^{-5}$ & 600 & 0.649 & 2.0 & 64 & 32 \\
\hline DNS2 & 0.0531 & 2.41 & $1.64 \times 10^{-5}$ & 1500 & 0.649 & 2.0 & 128 & 51 \\
\hline DNS3 & 0.134 & 2.41 & $1.64 \times 10^{-5}$ & 3900 & 0.649 & 2.0 & 256 & 81 \\
\hline DNS4 & 0.230 & 2.41 & $1.64 \times 10^{-5}$ & 6700 & 0.649 & 2.0 & 384 & 107 \\
\hline DNS5 & 0.339 & 2.41 & $1.64 \times 10^{-5}$ & 9800 & 0.649 & 2.0 & 512 & 129 \\
\hline DNS6 & 0.852 & 2.41 & $1.64 \times 10^{-5}$ & 25000 & 0.649 & 2.0 & 1024 & 205 \\
\hline
\end{tabular}

Finally, since $L_{x}=0.399 x_{o}$, the ratios $\overline{C^{* 2}} / C_{c}^{2}, \overline{u_{x}^{*} C^{*}} /\left(U_{c} C_{c}\right)$, and $\bar{\chi} /\left(U_{c} C_{c}^{2}\right)$ should only depend on the Reynolds number. In other words, after appropriate normalizations, the turbulent characteristics should only be determined by the Reynolds number.

\section{RESULTS}

\section{A. Simulation procedure}

The governing equations, Eqs. (6) and (25), are solved using the NGA code [27]. NGA is a three-dimensional, finite difference solver suitable for variable density, low Mach number, and laminar and turbulent flows. It solves the continuity, Navier-Stokes, and scalar transport equations in physical space, not in spectral space, while discretely conserving kinetic energy.

The initial velocity and scalar fields are randomly generated, following the method used by Eswaran and Pope [28]. The velocity fields are subject to the continuity constraint and conformed to a specified Passot-Pouquet energy spectrum [29]. The scalar fields are produced in a similar manner. A detailed explanation can be found in Ref. [30]. As mentioned in Sec. I, only unity Schmidt number simulations are considered in the present work. The Courant-Friedrichs-Lewy condition, $\mathrm{CFL} \leqslant 0.9$, has been imposed for all the simulations in the current paper.

The procedure of conducting the direct numerical simulation (DNS) in the current investigation can be summarized as follows:

(i) Find the centerline velocity $U_{c}$, centerline scalar $C_{c}$, and the axial location $x_{o}$ of the target experiment.

(ii) Use $U_{c} / x_{o}$ and $C_{c} / x_{o}$ to determine the source term for the DNS.

(iii) Use $x_{o}$ to determine the length of the DNS cubic box, $L_{x}$.

(iv) Perform the DNS in a triply periodic configuration with the target $v=D$.

As in our previous work, $L_{x}=0.399 x_{o}$ is used to match the integral length scale with a given target experiment; a detailed analysis can be found in Ref. [13].

Six DNS have been performed with $\mathrm{Sc}=1$, as shown in Table II, with the bounded cubic Hermite polynomial (BCH) scalar transport scheme [31]. The target experiment is a slightly heated turbulent air jet in Ref. [24]. However, since its Reynolds number is too high, we have applied lower $U_{c}$ values for our simulations to decrease the Reynolds numbers.

For each time-averaged quantity shown in this work, averaging uncertainty is presented as an error bar. This uncertainty quantification method is explained in detail in the Appendix (Sec. A). For the accurate examination of time-averaged values, the simulations have to run for a sufficiently long time. However, we could not run DNS6 long enough, because of its high computational cost. As a result, DNS6 has been omitted from analysis in Secs. IV B and IV D.

\section{B. Scaling coefficient}

In this section, we report the values of $\alpha_{1}, \alpha_{2}$, and $\alpha_{3}$ by using DNS1-5, for the three relations, Eqs. (31)-(33), in which time-averaged values of volume-averaged quantities are used in the place of 


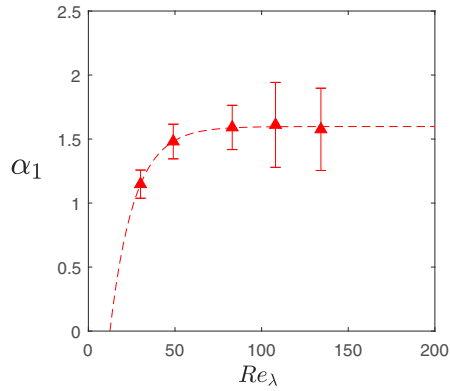

(a) Variance

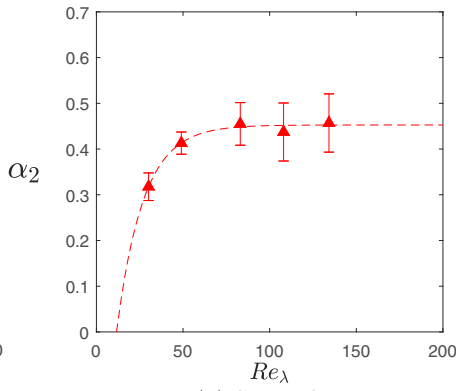

(b) Scalar flux

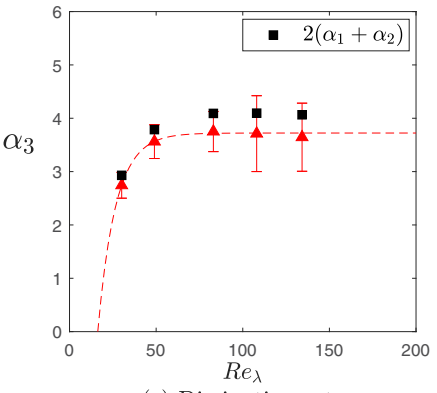

(c) Dissipation rate

FIG. 1. Scaling coefficients for the variance [(a), Eq. (31)], the scalar flux [(b), Eq. (32)], and the dissipation rate [(c), Eq. (33)] from DNS1-5. The dashed lines are weighted least-squares fits of a functional form $h\left(\operatorname{Re}_{\lambda}\right)=$ $a_{1}-a_{2} \exp \left(-a_{3} \operatorname{Re}_{\lambda}\right)$.

ensemble-averaged ones. This is justified because of the statistically homogeneous and statistically stationary nature of the flow. The results are plotted as a function of $\operatorname{Re}_{\lambda}$ in Fig. 1. The uncertainty tends to be larger for higher Reynolds number data, as it is more difficult to have a large number of samples due to the high computational cost. Nevertheless, we will focus on the average quantities for all data.

Each coefficient appears to approach a plateau rapidly, as the Reynolds number increases. The lack of an inertial range for low Reynolds number flows might be the cause for the low coefficient values. An exponential fitting is provided to estimate the high Reynolds number limit. The dashed lines are weighted least-squares fits of a functional form $h\left(\operatorname{Re}_{\lambda}\right)=a_{1}-a_{2} \exp \left(-a_{3} \operatorname{Re}_{\lambda}\right)$. It is found that

$$
\begin{gathered}
\alpha_{1} \rightarrow 1.60, \\
\alpha_{2} \rightarrow 0.453, \\
\alpha_{3} \rightarrow 3.72
\end{gathered}
$$

As stated in Sec. III C, the three coefficients are related by the scalar variance equation under statistical stationarity [Eq. (27)]. More precisely, it can be deduced that

$$
\alpha_{3} \approx 2\left(\alpha_{1}+\alpha_{2}\right)
$$

Figure 1(c) shows $2\left(\alpha_{1}+\alpha_{2}\right)$. There is a small difference between $\alpha_{3}$ and $2\left(\alpha_{1}+\alpha_{2}\right)$, which is the inevitable consequence of numerical dissipation of the scalar transport scheme. Figure 2 displays such dissipation defined as $\alpha_{\text {num }} \equiv 2\left(\alpha_{1}+\alpha_{2}\right)-\alpha_{3}$ for DNS1-5, which increases with the Reynolds number. The ratio of numerical to physical dissipation increases from about $7 \%$ to about $10 \%$.

\section{Instantaneous mixing structures}

To study the impact of the source terms, three scalars were transported simultaneously for all simulations: the first scalar with the original jet centerline (JC) source terms, $\frac{U_{c}}{x_{o}} C^{*}+\frac{C_{c}}{x_{o}} u_{x}^{*}$; the second scalar with only the linear scalar (LS) term with the target variance $\sigma_{t}^{2}=0.001, \frac{U_{c}}{x_{o}} C^{*}$; and the third scalar with only the mean gradient (MG) term, $\frac{C_{c}}{x_{o}} u_{x}^{*}$. The contours of these three scalar fields on a cross section in the middle of the simulation cubic box from DNS2 are shown in Fig. 3. Each plot uses 10 contour levels from its own minimum value (dark blue) to maximum value (bright yellow). 


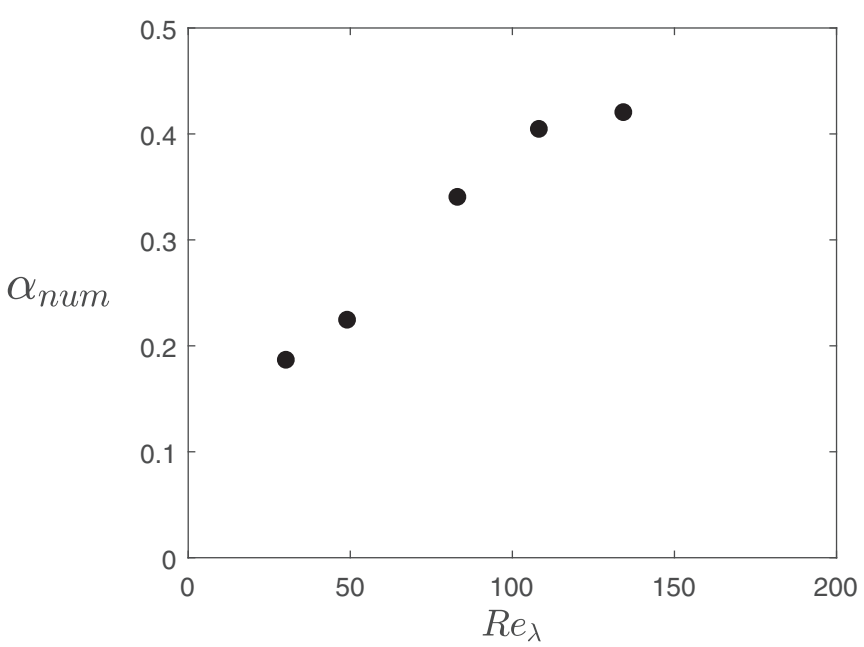

FIG. 2. Numerical dissipation defined as $\alpha_{\text {num }} \equiv 2\left(\alpha_{1}+\alpha_{2}\right)-\alpha_{3}$ for DNS1-5.

The mixing structures are homogeneous for all plots, as expected. Although the three forcing methods produce different scalar statistics, as will be shown in Sec. IV D, it is difficult to identify visually the difference from the contour plots.

The JC plots are highly similar to the MG plots, which is to be expected because one of the terms in the JC forcing method is a mean gradient term, $\frac{C_{c}}{x_{o}} u_{x}^{*}$. Since this term is shared by both JC and MG, there must be similarities in any instantaneous JC and MG scalar fields. However, they are not
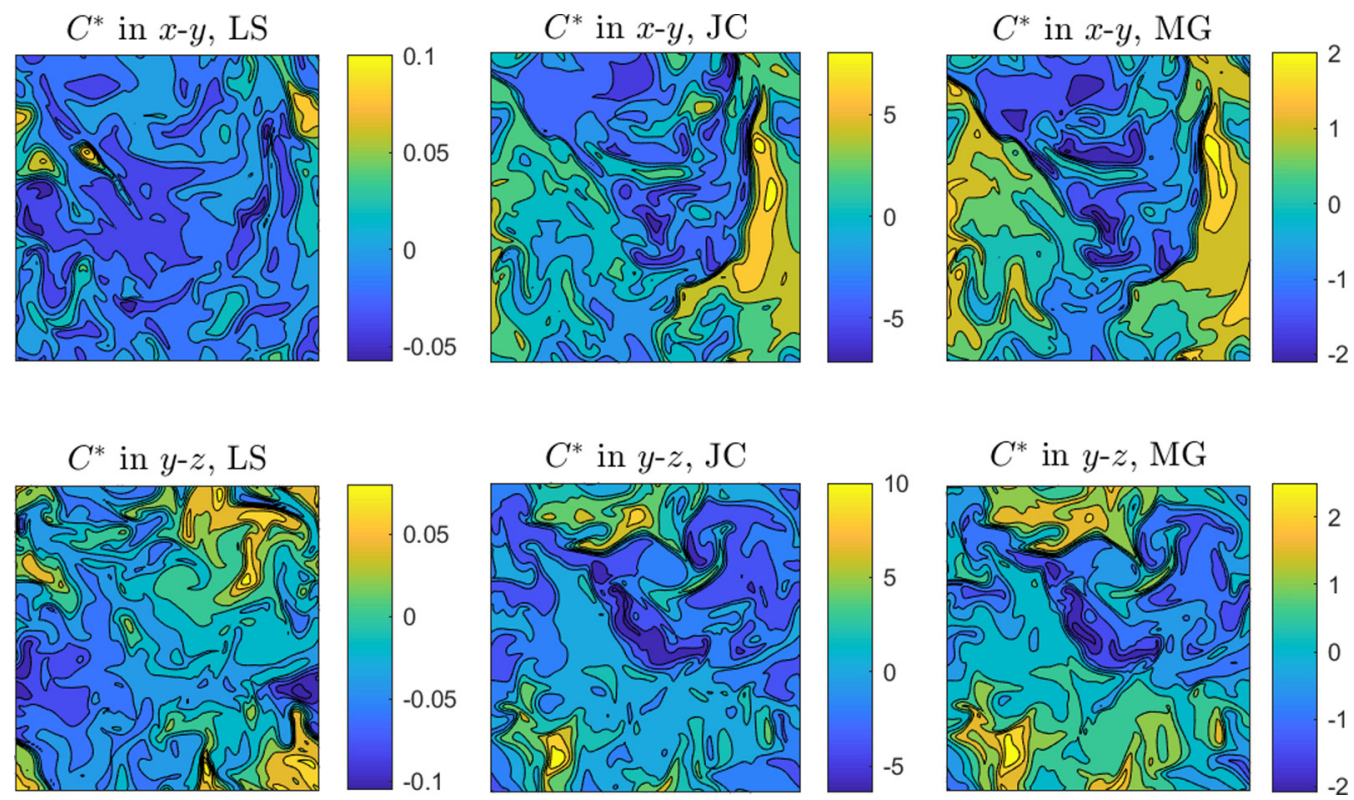

FIG. 3. Contours of scalar fields from DNS2 in $x y$ (top) and $y z$ (bottom) planes with three different forcing methods: the linear scalar (LS) forcing (left), the jet centerline (JC) forcing (middle), and the mean gradient (MG) forcing (right). 


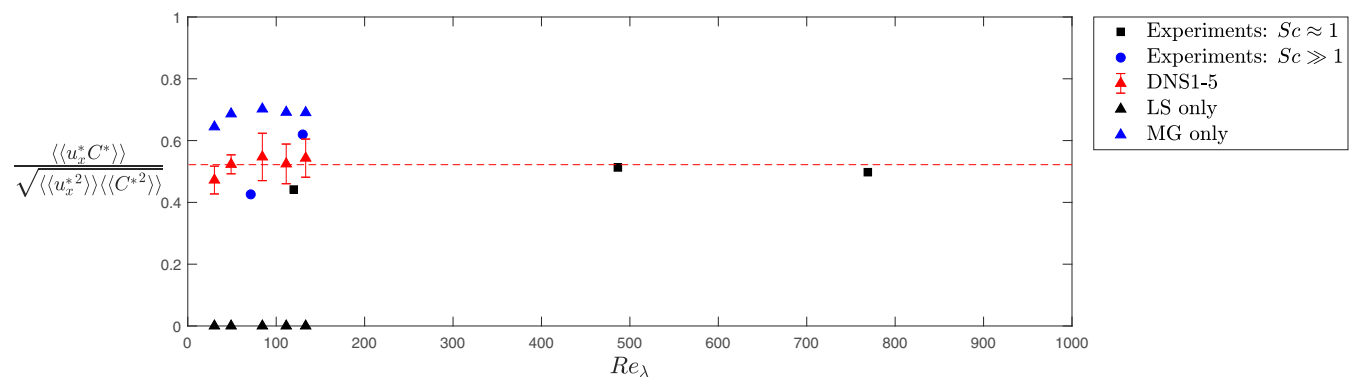

FIG. 4. Normalized scalar flux $\frac{\left\langle\left\langle u_{x}^{*} C^{*}\right\rangle\right\rangle}{\sqrt{\left\langle\left\langle u_{x}^{* 2}\right\rangle\right\rangle\left\langle\left\langle C^{* 2}\right\rangle\right\rangle}}$ and its corresponding experimental quantity. Round jet experiments with $\mathrm{Sc} \approx 1$ are from Anderson and Bremhorst [23], Darisse et al. [24], and Chevray and Tutu [32], from low to high Reynolds numbers. Round jet experiments with Sc $\gg 1$ are from Webster et al. [21] and Antoine et al. [33], from low to high Reynolds numbers. The figure displays DNS1-5 results with the original forcing terms, $\frac{U_{c}}{x_{o}} C^{*}+\frac{C_{c}}{x_{o}} u_{x}^{*}$ (red triangles); with only the linear scalar (LS) term, $\frac{U_{c}}{x_{o}} C^{*}$ (black triangles); and with only the mean gradient (MG) term, $\frac{C_{c}}{x_{o}} u_{x}^{*}$ (blue triangles). The mean of DNS1-5 values with the original forcing terms is 0.52 , shown as the dashed red line.

exactly the same, because there is another term in $\mathrm{JC}$ forcing, the linear scalar term. This additional LS term leads to a larger scalar variance compared to that of MG forcing.

For the scalars in LS plots, it is difficult to compare the magnitude against others, as the variance is imposed to be $\sigma_{t}^{2}=0.001$ in LS forcing. As compared to MG plots, the LS ones are much less similar to JC plots; there is much less correlation between the LS fields and the JC fields.

\section{Validation against experimental data: Scalar flux}

Normalized scalar flux quantities, $\left\langle\left\langle u_{x}^{*} C^{*}\right\rangle\right\rangle / \sqrt{\left\langle\left\langle u_{x}^{* 2}\right\rangle\right\rangle\left\langle\left\langle C^{* 2}\right\rangle\right\rangle}$, have been calculated from simulations and compared against experiments. The equivalent experimental value is

$$
\left.\frac{\left\langle u_{x}^{\prime} C^{\prime}\right\rangle_{t}}{\sqrt{\left\langle u_{x}^{\prime 2}\right\rangle_{t}\left\langle C^{\prime 2}\right\rangle_{t}}}\right|_{x=x_{o}},
$$

where $\langle\cdot\rangle_{t}$ denotes time averaging. The comparison is shown in Fig. 4.

Unity Schmidt number experiments are from works by Anderson and Bremhorst [23], Darisse et al. [24], and Chevray and Tutu [32]. High Schmidt number experiments from the works of Webster et al. [21] and Antoine et al. [33] are also listed for completeness, because the range of scalar flux values appears independent of Schmidt numbers. $\mathrm{Re}_{\lambda}=1.3 \sqrt{\mathrm{Re}_{D}}$ has been applied to the experiments to convert $\operatorname{Re}_{D}$ to $\operatorname{Re}_{\lambda}$ [26]. There is some scatter in the published values ranging from 0.4 to 0.6 for both unity and high Schmidt number experiments. However, there are no apparent trends.

To study the impact of the source terms, three cases have been performed with the same velocity field. In other words, three scalars were transported simultaneously for all simulations: the first scalar with the original source terms, $\frac{U_{c}}{x_{o}} C^{*}+\frac{C_{c}}{x_{o}} u_{x}^{*}$; the second scalar with only the linear scalar (LS) term with the target variance $\sigma_{t}=0.001, \frac{U_{c}}{x_{o}} C^{*}$; and the third scalar with only the mean gradient (MG) term, $\frac{C_{c}}{x_{o}} u_{x}^{*}$. The error bars are only shown for the flux with the original source terms (red triangles) and were estimated using the numerator $\left\langle\left\langle u_{x}^{*} C^{*}\right\rangle\right\rangle$ only. Once again, the simulations in the current study have been performed with $\mathrm{Sc}=1$.

The scalar flux values from the current study seem fairly constant, independently of the Reynolds number. In the discussion of scaling coefficients in Sec. IV B, the effect of Reynolds number is already diminished at $\operatorname{Re}_{\lambda} \geqslant 50$. It is very likely that the temporal mean of volume-averaged 


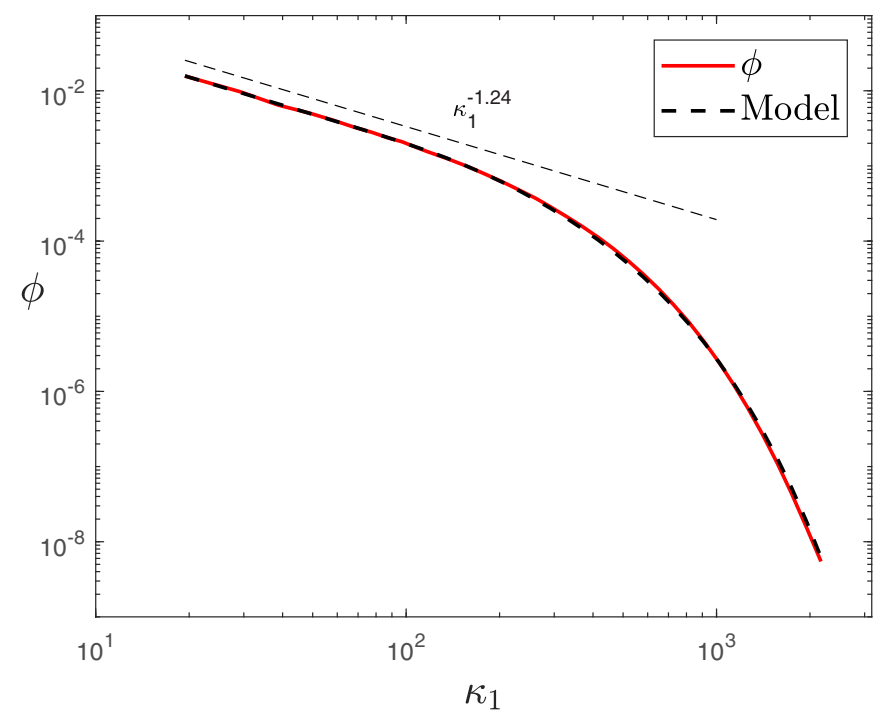

FIG. 5. Scalar energy spectrum: $\phi\left(\kappa_{1}\right)$ computed from DNS5 at $\operatorname{Re}_{\lambda}=129$, and its least-squares fit result with model spectrum, Eq. (40).

turbulent quantities is quite constant for $\operatorname{Re}_{\lambda} \geqslant 50$. Therefore, the effect of Reynolds number on normalized scalar flux must also be minimal for higher Reynolds numbers.

The mean of four scalar flux values with the original source terms, $\frac{U_{c}}{x_{\rho}} C^{*}+\frac{C_{c}}{x_{o}} u_{x}^{*}$, is about 0.52 , which is expressed with the red dashed line in Fig. 4. This value lies within the range of the reported experimental values. In contrast, the scalar flux $\left\langle\left\langle u_{x}^{*} C^{*}\right\rangle\right\rangle$ is zero for LS forcing, because there is no term in the advection-diffusion equation that produces a correlation between the velocity and the scalar. When the DNS is performed only with the MG term, the scalar flux is systematically larger than the largest experimental values.

\section{E. Validation against experimental data: Scaling exponent of energy spectra}

A scalar energy spectrum can be easily computed from the triply periodic DNS. The onedimensional energy spectrum, $\phi\left(\kappa_{1}\right)$, is defined as

$$
\int_{0}^{\infty} \phi\left(\kappa_{1}\right) d \kappa_{1}=\frac{1}{2}\left\langle C^{* 2}\right\rangle,
$$

where $\kappa_{1}$ is the wave number in the longitudinal direction. $\phi\left(\kappa_{1}\right)$ is the Fourier transform of the spatial correlation function $\left\langle C^{*}(\boldsymbol{x}) \cdot C^{*}\left(\boldsymbol{x}+\boldsymbol{r}_{\mathbf{1}}\right)\right\rangle$, where $\boldsymbol{r}_{\mathbf{1}}$ is a vector in the longitudinal direction. An example of scalar energy spectra is shown in Fig. 5. It is computed from DNS5 at $\operatorname{Re}_{\lambda}=129$.

Our interest is to determine the scaling exponent $n$ for the relation $\phi \sim \kappa^{-n}$ in the inertialconvective subrange and compare it against experiments. We use the following model spectrum:

$$
\hat{\phi}\left(\kappa_{1}\right)=B_{1} \kappa_{1}^{-n} \exp \left\{-B_{2}\left(\left[\left(\eta \kappa_{1}\right)^{4}+B_{3}^{4}\right]^{\frac{1}{4}}-B_{3}\right)\right\},
$$

where $B_{1}, B_{2}$, and $B_{3}$ are constants, and $\eta=\left(v^{3} / \varepsilon\right)^{1 / 4}$ is the Kolmogorov length scale. This form of a function was used by Lee et al. to model a scalar spectrum [38]. The exponential part for the dissipation range is adopted from the kinetic energy model spectrum of Pope [16]. A least-squares fit is used over the entire spectrum with Eq. (40) to determine $B_{1}, B_{2}, B_{3}$, and $n$. For the example shown in Fig. 5, $n$ is found to be 1.24 with this method. All of the fitting results are shown in Table III. 
TABLE III. Least-squares fit results.

\begin{tabular}{lcccl}
\hline \hline & $B_{1}$ & $B_{2}$ & $B_{3}$ & $n$ \\
\hline SCL1 & 0.744 & 4.86 & $1.21 \times 10^{-4}$ & 0.964 \\
SCL2 & 0.843 & 3.85 & 0.0788 & 1.03 \\
SCL3 & 0.492 & 5.37 & 0.106 & 1.15 \\
SCL4 & 0.184 & 5.13 & 0.120 & 1.20 \\
SCL5 & 0.650 & 5.40 & 0.110 & 1.24 \\
SCL6 & 0.345 & 5.30 & 0.090 & 1.33 \\
\hline \hline
\end{tabular}

The $n$ values computed from DNS1-6 are displayed in Fig. 6. They show a clear trend of increasing scaling exponents with increasing $\mathrm{Re}_{\lambda}$. Experimental values of round jets are taken from Refs. [34,35]. For the spectrum in Ref. [35], we applied the same least-squares fit to find the scaling exponent. Although our simulations do not match the $\operatorname{Re}_{\lambda}$ of experiments presented in this work exactly, the result from DNS6 is comparable to the two experimental values.

Scaling exponents from various other shear flows [36] are also shown in Fig. 6 for an additional comparison. The dashed line is a fit provided by Ref. [37] for these shear flows. Although it is difficult to conclude from this comparison that round jets display the same scalar energy spectra as other shear flows, it should be noted that the $n$ values from our simulations, jet experiments, and other shear flows are along the similar increase curve.

\section{DISCUSSION}

In this section, we examine the effects of the velocity forcing scheme on the statistics of the scalar field. The numerical computations presented in the current study apply the jet centerline (JC) velocity forcing on velocity fields and the JC scalar forcing on the scalar fields.

\section{A. Simulations with different velocity forcing terms}

The forcing term for the JC velocity method is anisotropic and linear with the velocity components, as shown in Eq. (10). We will denote the forcing coefficient $U_{c} / 2 x_{o}$ as $A$. Then, the forcing terms in the original JC velocity method can be expressed as

$$
\begin{aligned}
\boldsymbol{f}_{u} & =f_{x} u_{x}^{*} \hat{\mathbf{i}}+f_{y} u_{y}^{*} \hat{\mathbf{j}}+f_{z} u_{z}^{*} \hat{\mathbf{k}} \\
& =2 A u_{x}^{*} \hat{\mathbf{i}}+A u_{y}^{*} \hat{\mathbf{j}}+A u_{z}^{*} \hat{\mathbf{k}} .
\end{aligned}
$$

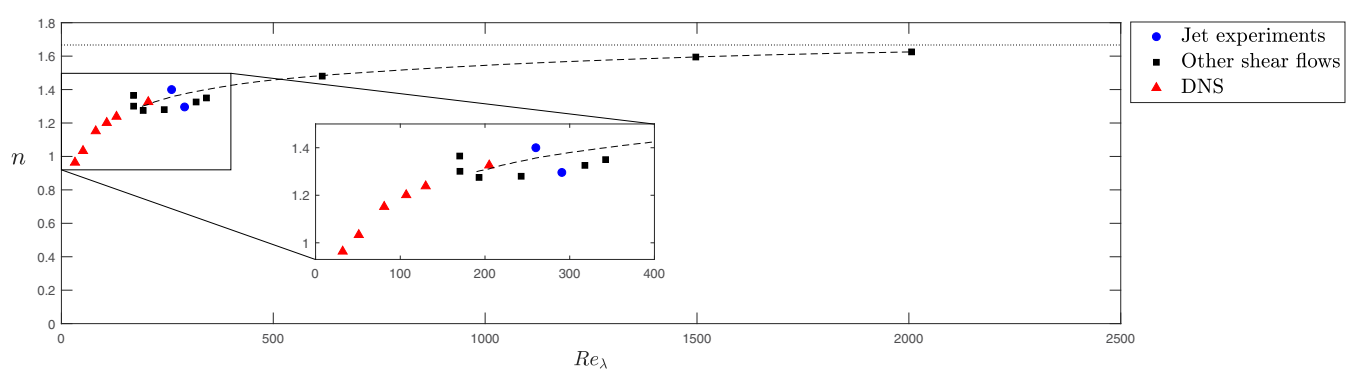

FIG. 6. Scaling exponent $n$ for the scalar energy spectra, $\phi \sim \kappa^{-n}$, calculated from DNS1-6. Experimental values are taken from two round jets [34,35] and other shear flows [36]. The dashed line is a fit provided by Ref. [37]. The dash-dotted line shows $n=5 / 3$. 
TABLE IV. Forcing coefficients for the linear velocity forcing terms for simulations with $A=U_{c} / 2 x_{o}$.

\begin{tabular}{lcccccc}
\hline \hline & $f_{x}$ & $f_{y}$ & $f_{z}$ & $\operatorname{Re}_{\lambda}$ & $r_{\chi}$ & $2 \frac{U_{c}}{x_{o}} \frac{\langle\langle\varepsilon\rangle\rangle}{\langle\langle k\rangle\rangle}$ \\
\hline DNS1 & $2 A$ & $A$ & $A$ & 32 & 1.5 & 1.2 \\
Iso1 & $A$ & $A$ & $A$ & 22 & 1.8 & 2.1 \\
Iso2 & $4 A$ & $A$ & $A$ & 49 & 1.3 & 0.76 \\
Mag1 & $A$ & $A / 2$ & $A / 2$ & 22 & 2.0 & 2.6 \\
Mag2 & $4 A$ & $2 A$ & $2 A$ & 43 & 1.2 & 0.63 \\
\hline \hline
\end{tabular}

Additional DNS have been performed with different coefficients for the linear velocity forcing terms, as shown in Table IV. Each simulation still uses the same JC scalar forcing.

The baseline case is DNS1 from Table II. Iso1 is isotropic, while Iso2 imposes a stronger anisotropy than DNS1. Mag1-2 use the same $2: 1: 1$ ratio for the forcing coefficients, but Mag 1 is smaller and Mag2 is larger in forcing magnitudes than DNS1. Iso1 and Mag1 are found to be unstable; their statistical values, such as volume-averaged variance and dissipation, increase exponentially over time. The stability issue will be discussed in Sec. V D.

\section{B. Timescale ratio}

Let us define the timescale ratio as

$$
r_{\chi} \equiv \frac{\bar{\chi} / \overline{C^{* 2}}}{\bar{\varepsilon} / \bar{k}} .
$$

For simulations with the proposed JC scalar forcing, we can deduce from Eqs. (31) and (33) in Sec. III C that

$$
\frac{\bar{\chi}}{\overline{C^{* 2}}}=\frac{\alpha_{3}}{\alpha_{1}} \frac{U_{c}}{x_{o}}
$$

Also, for the JC velocity forcing, we can find from Eqs. (11) and (12) that

$$
\frac{\bar{\varepsilon}}{\bar{k}}=\left(1+\frac{\left\langle\left\langle u_{x}^{* 2}\right\rangle\right\rangle}{\left\langle\left\langle\boldsymbol{u}^{* 2}\right\rangle\right\rangle}\right) \frac{U_{c}}{x_{o}},
$$

with the assumption that the ensemble-averaged quantities are equal to their expected values.

The timescale ratio, $r_{\chi}$, for the simulations with the original JC velocity and the proposed JC scalar forcing will be

$$
r_{\chi}=\frac{\alpha_{3} / \alpha_{1}}{1+\frac{\left\langle\left\langle u_{x}^{* 2}\right\rangle\right\rangle}{\left\langle\left\langle\boldsymbol{u}^{22}\right\rangle\right\rangle}} .
$$

From the exponential fits in Sec. IV B, $r_{\chi}$ should be around 1.5 for any $\operatorname{Re}_{\lambda}$ larger than 25 .

The ratios $r_{\chi}$ from DNS1-5, Iso1-2, and Mag1-2 are displayed in Fig. 7. For the computation of $r_{\chi}$, the temporal mean of $\left(\langle\chi\rangle /\left\langle C^{* 2}\right\rangle\right) /(\langle\varepsilon\rangle /\langle k\rangle)$ has been used, where $\langle\cdot\rangle$ denotes a volumeaveraged quantity at one sample time.

As expected from Eq. (46), $r_{\chi}$ of DNS1-5 is around 1.5. The ratios $r_{\chi}$ of Iso2 and Mag2 are smaller than those of DNS1-5 by about $18 \%$ and $25 \%$, respectively. Nevertheless, the ratio of scalar to velocity timescales is fairly independent of the velocity forcing scheme (both magnitude and anisotropy). 


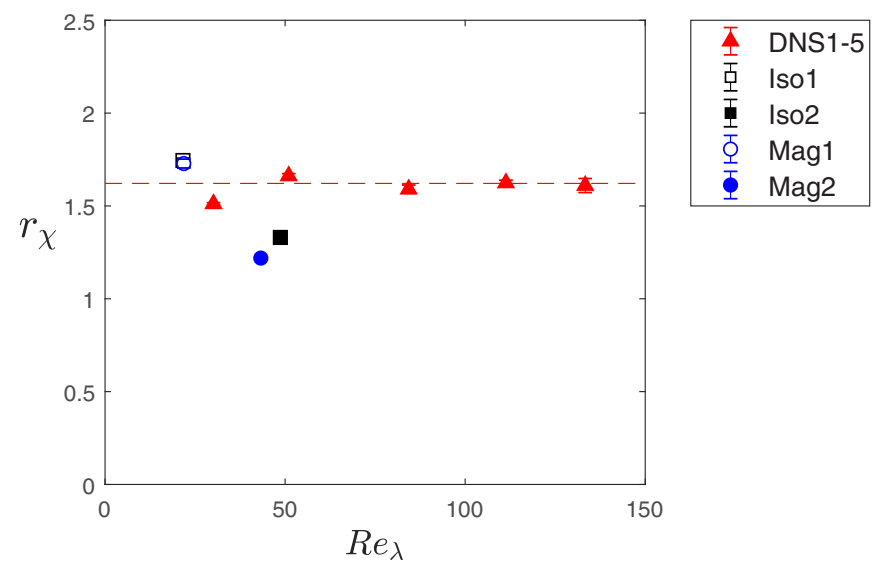

FIG. 7. Timescale ratio $r_{\chi}$ [Eq. (43)] from DNS1-5, Iso1-2, and Mag1-2. The mean of DNS1-5 values is shown as the dashed red line.

\section{Effect of velocity forcing on scalar flux}

Normalized scalar flux quantities, $\left\langle\left\langle u_{x}^{*} C^{*}\right\rangle\right\rangle / \sqrt{\left\langle\left\langle u_{x}^{* 2}\right\rangle\right\rangle\left\langle\left\langle C^{* 2}\right\rangle\right\rangle}$, have been computed from DNS15, Iso1-2, and Mag1-2, as shown in Fig. 8. The flux values of Iso2 and Mag2 are larger than those of DNS1-5, with a maximum deviation of about $18 \%$ for Mag2. In clear contrast, $\left\langle\left\langle u_{x}^{*} C^{*}\right\rangle\right\rangle / \sqrt{\left\langle\left\langle u_{x}^{* 2}\right\rangle\right\rangle\left\langle\left\langle C^{* 2}\right\rangle\right\rangle}$ of Iso1 and Mag1 are essentially zero, which indicates that velocity and scalar fields remain uncorrelated, despite the MG term in the transport equation. This phenomenon is actually caused by the instability of Iso1 and Mag1 simulations and is discussed next.

\section{Stability of scalar equation}

The stability of our simulation system can be examined by analyzing the scalar variance equation. Using Eq. (43), the equation for the volume-averaged scalar variance $\left\langle C^{* 2}\right\rangle$ can be expressed as

$$
\frac{d\left\langle C^{* 2}\right\rangle}{d t}=-\frac{\langle\varepsilon\rangle}{\langle k\rangle}\left(r_{\chi}-2 \frac{U_{c}}{x_{o}} \frac{\langle k\rangle}{\langle\varepsilon\rangle}\right)\left\langle C^{* 2}\right\rangle+2 \frac{C_{c}}{x_{o}}\left\langle u_{x}^{*} C^{*}\right\rangle .
$$

If $r_{\chi}>2 \frac{U_{c}}{x_{o}} \frac{\langle k\rangle}{\langle\varepsilon\rangle}$, the solution is stable; the two terms on the right-hand side (RHS) become balanced, and the system finds a statistically stationary state. However, if $r_{\chi}<2 \frac{U_{c}}{x_{o}} \frac{\langle k\rangle}{\langle\varepsilon\rangle}$, the solution is unstable; the scalar variance keeps increasing and does not reach a statistically stationary state.

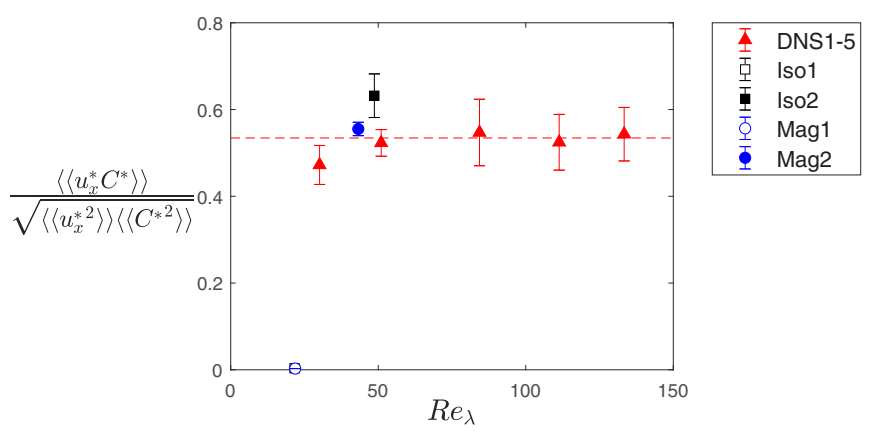

FIG. 8. Normalized scalar flux from DNS1-5, Iso1-2, and Mag1-2. The mean of DNS1-5 values is shown as the dashed red line. 


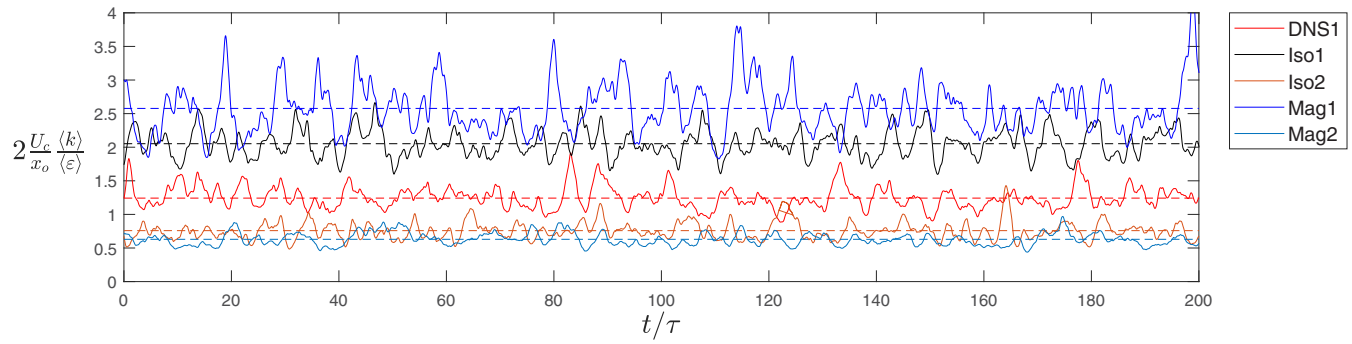

FIG. 9. Volume-averaged ratio, $2 \frac{U_{c}}{x_{o}} \frac{\langle k\rangle}{\langle\varepsilon\rangle}$, from DNS1, Iso1-2, and Mag1-2, plotted as a function of the time normalized by the eddy timescale, $\tau=k_{o} / \varepsilon_{o}$. The horizontal dashed line indicates the time-averaged value of each simulation.

Figure 9 displays the ratios of volume-averages, $2 \frac{U_{c}}{x_{o}} \frac{\langle k\rangle}{\langle\varepsilon\rangle}$, from DNS1, Iso1-2, and Mag1-2, plotted as a function of normalized time. As a reminder, for passive scalars, this ratio is only a result of the velocity forcing scheme and is not influenced by the scalar field. Table IV reports the time averages of $r_{\chi}$ and $2 \frac{U_{c}}{x_{o}}\left\langle\frac{\langle k\rangle}{\langle\varepsilon\rangle}\right.$ for each simulation.

As expected from Eq. (47), for stable simulations, DNS1, Iso2, and Mag2, the time-averaged value of $2 \frac{U_{c}}{x_{o}} \frac{\langle k\rangle}{\langle\varepsilon\rangle}$ is smaller than its respective $r_{\chi}$. On the other hand, for unstable simulations, Iso1 and Mag1, the time-averaged value of $2 \frac{U_{c}}{x_{o}} \frac{\langle k\rangle}{\langle\varepsilon\rangle}$ is larger than its respective $r_{\chi}$. For unstable Iso1 and Mag1, the velocity-scalar correlation cannot be developed, as shown in Sec. V C, because the scalar field cannot reach a statistically stationary state.

We have found that the velocity forcing affects the scalar statistics and the stability of the simulations. It also shows the need for an appropriate relation between the velocity and scalar forcings. Simulations with the proposed JC scalar forcing must use the JC velocity forcing, in order to produce the proper turbulence of a round jet on the centerline. This inherent relation ensures that the simulations are stable and produce the correct scalar turbulence.

\section{CONCLUSION}

The forcing method derived in this work is based on the physical properties of a turbulent round jet, which are applied to the scalar transport equation. A normalization on the scalar has also been applied to derive the source term to be used in a triply periodic box. The derivation result is a combination of two previously existing methods, namely mean gradient (MG) and linear scalar (LS).

A dimensional analysis was introduced to seek relations between the inputs and outputs of our simulations with jet centerline (JC) velocity and JC scalar forcing. It was found that normalized scalar statistics, such as variance, flux, and dissipation rate, should only be a function of Reynolds number. Our simulation results indicate that such quantities approach constant values as the Reynolds number increases.

Normalized scalar flux quantities and scaling exponents of scalar energy spectra were compared against experiments. Unfortunately, only a small amount of data is available in the literature, and some scatter among the experimental values exists. There is a difficulty in the comparison, because of the small amount of available data in the literature and the scatter among the experimental values. Nevertheless, the simulations results are comparable to the round jet measurements.

The effects of velocity forcing schemes on the scalar fields were also investigated by altering the velocity forcing coefficients while maintaining the same JC scalar forcing. It was observed that velocity forcing had a slight influence on the resulting timescale ratio $r_{\chi}$ and scalar flux. More importantly, however, changing velocity forcing terms may result in unstable scalar fields even 
under the same scalar forcing. This finding shows that a scalar forcing needs to be properly derived in relation to this particular velocity forcing, at least.

The current study devised the scalar forcing technique that replicates the mixing nature in a practical flow. It proves the possibility of creating a realistic mixing environment in a simple 3D periodic domain instead of computing an entire flow. The mathematical methodology is not limited to turbulent round jet centerlines and should be applied to different flow configurations in future studies.

\section{ACKNOWLEDGMENTS}

The authors gratefully acknowledge funding from the National Science Foundation (CBET No.1512771 and No. 1761690) and from the Air Force Office of Scientific Research (No. FA955016-1-0510) under the supervision of Dr. Chiping Li. In addition, K.J.R. acknowledges the financial support from the Samsung Scholarship Foundation.

\section{APPENDIX: UNCERTAINTY QUANTIFICATION}

\section{Definitions}

Suppose there are $N$ time-sequential samples available, modeled as a stationary ergodic random process $x_{i}$ sampled at every $\Delta t$. The autocorrelation function for this random process is defined as

$$
\rho(k) \equiv \frac{E\left[\left(x_{i}-\mu\right)\left(x_{i+k}-\mu\right)\right]}{\sigma^{2}},
$$

where $k$ is the lag, $\mu$ is the expected mean of $x_{i}$, and $\sigma^{2}$ is the variance, $E\left[\left(x_{i}-\mu\right)^{2}\right]$.

These samples are then divided into $p$ number of nonoverlapping segments with length $n=N / p$. A mean value, $\hat{s}_{j}$, is calculated for each $j$ th segment:

$$
\hat{s}_{j}=\frac{1}{n} \sum_{i=1+n(j-1)}^{n j} x_{i}
$$

The set of mean values from the segments, $\left\{\hat{s}_{j}\right\}$, can also be considered as the elements of a random process. The expected mean of $\hat{s}_{j}$ is $\mu$. Let us define the expected averaging error as

$$
\delta_{n}^{2} \equiv E\left[\left(\hat{s}_{j}-\mu\right)^{2}\right]
$$

The goal of this uncertainty quantification is to estimate $\delta_{n}^{2}$ when $n \rightarrow N$.

\section{Modeling}

According to Ref. [39],

$$
\delta_{n}^{2}=\frac{\sigma^{2}}{n}\left[1+2 \sum_{k=1}^{n-1}\left(1-\frac{k}{n}\right) \rho(k)\right] .
$$

Rearranging the terms, we obtain

$$
\delta_{n}^{2}=\frac{1}{n}\left[\sigma^{2}+2 \sigma^{2} \sum_{k=1}^{n-1} \rho(k)\right]-\frac{1}{n^{2}}\left[2 \sigma^{2} \sum_{k=1}^{n-1} k \rho(k)\right] .
$$

Inspired by the form of Eq. (A5), we suggest modeling the averaging error as

$$
\delta_{n}^{2} \approx f(n ; \boldsymbol{B})=\frac{B_{1}}{n}+\frac{B_{2}}{n^{2}},
$$




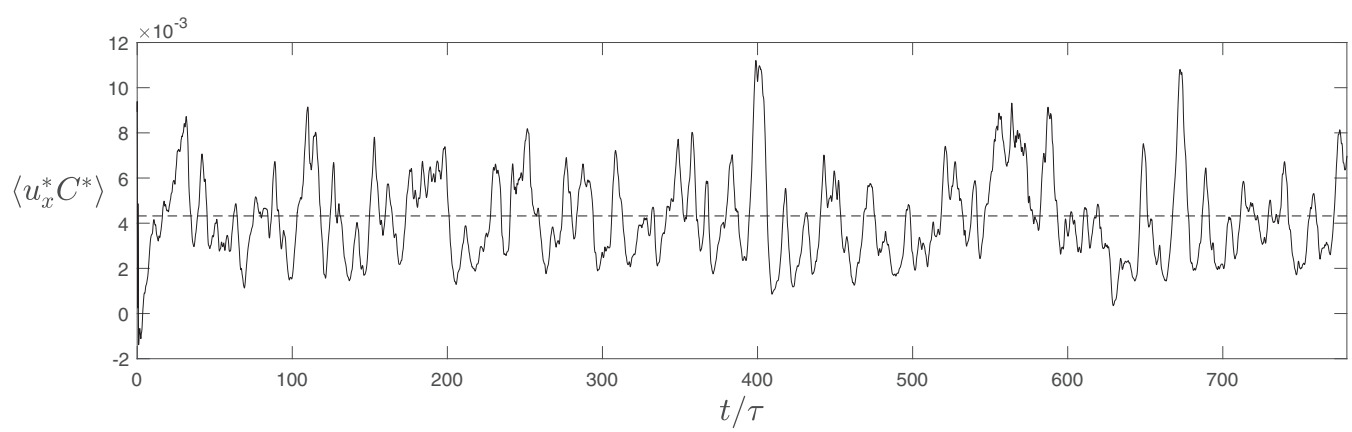

FIG. 10. Volume-averaged scalar flux, $\left\langle u_{x}^{*} C^{*}\right\rangle$, from DNS2 plotted as a function of the time normalized by the eddy timescale, $\tau$. The horizontal dashed line indicates its time-averaged value.

where $\boldsymbol{B}=\left[B_{1}, B_{2}\right]$ is a vector of parameters. This expression is exact in the limit of $n \Delta t$ sufficiently larger than the integral timescale.

\section{Calculation}

In practice, we compute the averaging error for segments of length $n$ as

$$
\hat{\delta}^{2}(n)=\frac{1}{p} \sum_{j=1}^{p}\left[\hat{s}_{j}(n)-\hat{\mu}\right]^{2},
$$

where

$$
\hat{\mu}=\frac{1}{N} \sum_{i=1}^{N} x_{i} .
$$

Then, a least-squares fit is used over $\hat{\delta}^{2}(n)$ with Eq. (A6) to determine $\boldsymbol{B}$ by solving

$$
\boldsymbol{B}=\arg \min _{\boldsymbol{B}} \sum_{n=n_{k}}^{N / 2}\left[\hat{\delta}^{2}(n)-f(n ; \boldsymbol{B})\right]^{2} .
$$

Theoretically, the minimum number of segments is $p=2$; thus, the maximum segment length is $N / 2$. As mentioned previously, the minimum segment length must be sufficiently large for Eq. (A6) to be valid. We suggest varying $n_{k}$, determining $\boldsymbol{B}$ for each $n_{k}$ value, and finally finding the maximum $f(N)$ :

$$
\hat{\delta}_{N}^{2}=\max _{n_{k}} f\left(N ;\left.\boldsymbol{B}\right|_{n_{k}}\right) .
$$

Because it is the most conservative choice, we will use this maximum $f(N ; \boldsymbol{B})$ as the final estimation for $\delta_{n}^{2}$ as $n \rightarrow N$.

\section{Example}

The volume-averaged scalar flux, $\left\langle u_{x}^{*} C^{*}\right\rangle$, has been computed from DNS2, and is shown in Fig. 10, as a function of normalized time. In this example, the transient period is about $15 \tau$. The dashed line indicates the time-averaged value of the entire data. The algorithm introduced in the previous section is applied to this data. 


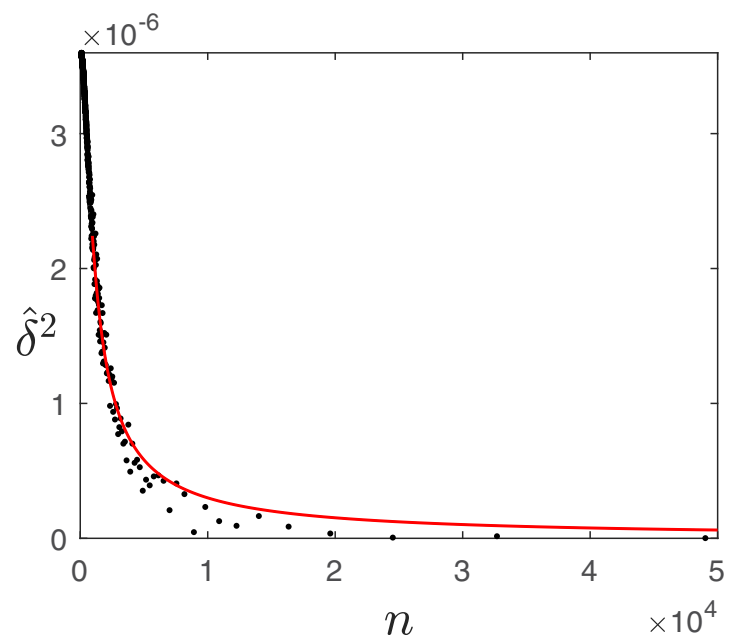

FIG. 11. Averaging error $\hat{\delta}^{2}$ as a function of $n$, for $\left\langle u_{x}^{*} C^{*}\right\rangle$ data presented in Fig. 10. The solid line represents the least-squares fit result with $\hat{\delta}^{2}$ for $n \geqslant 920: \hat{\delta}^{2}=0.00309 / n-0.911 / n^{2}$.

$\hat{\delta}^{2}(n)$ from Eq. (A7) is shown in Fig. 11. Then, $f\left(N ;\left.\boldsymbol{B}\right|_{n_{k}}\right)$ from Eq. (A10) is computed as a function of $n_{k}$ and displayed in Fig. 12. In this example,

$$
\arg \max _{n_{k}} f\left(N ;\left.\boldsymbol{B}\right|_{n_{k}}\right)=920 .
$$

The corresponding fit result, $f\left(n ;\left.\boldsymbol{B}\right|_{n_{k}=920}\right)$ for $n \geqslant 920$, is shown in Fig. 11. Finally, the estimated averaging error is

$$
\begin{gathered}
\hat{\delta}_{N}^{2}=3.56 \times 10^{-8}, \\
\sqrt{\hat{\delta}_{N}^{2}}=1.87 \times 10^{-4} .
\end{gathered}
$$

$\sqrt{\hat{\delta}_{N}^{2}}$ is about $4.32 \%$ of the mean value $\mu=0.00437$.

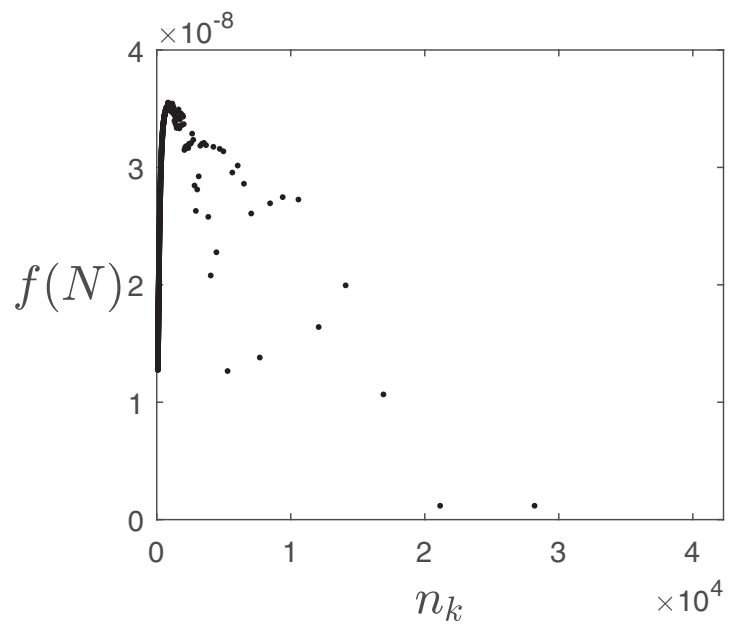

FIG. 12. $f\left(N ;\left.\boldsymbol{B}\right|_{n_{k}}\right)$ from Eq. (A10) is computed as a function of $n_{k}$. 


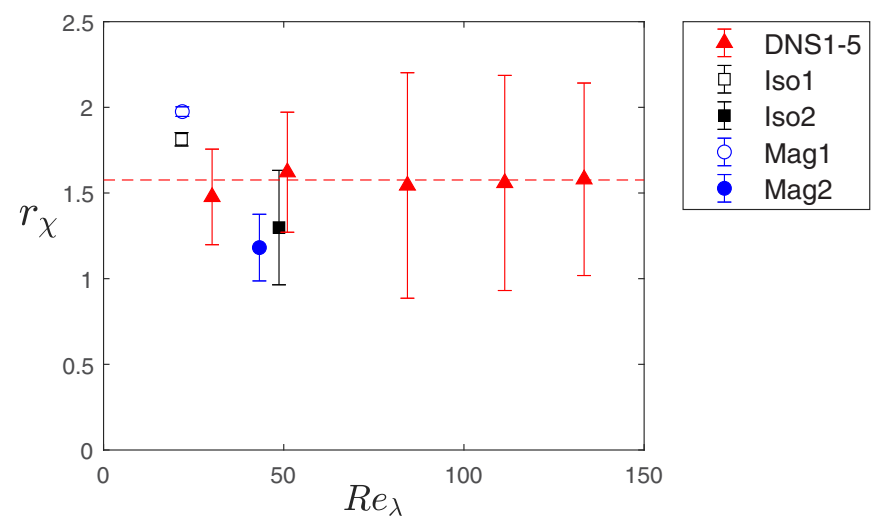

FIG. 13. The plot for timescale ratio [Eq. (43)] reproduced using summations of uncertainties for four parameters, $\langle\chi\rangle,\left\langle C^{* 2}\right\rangle,\langle\varepsilon\rangle$, and $\langle k\rangle$.

The same procedure has been repeated for each data point presented throughout the paper. The error bar indicates $\pm \sqrt{\hat{\delta}_{N}^{2}}$.

\section{Uncertainty quantification for ratios of parameters}

The timescale ratio $r_{\chi}$, defined in Eq. (43), is a ratio of four parameters. In Sec. V B, we computed $\left(\langle\chi\rangle /\left\langle C^{* 2}\right\rangle\right) /(\langle\varepsilon\rangle /\langle k\rangle)$ at each time step, and calculated the associated uncertainty.

Another method is to compute the uncertainties associated with four parameters separately and add them. For example, we can compute $\langle\chi\rangle,\left\langle C^{* 2}\right\rangle,\langle\varepsilon\rangle$, and $\langle k\rangle$ at each time step and calculate the uncertainty for each parameter. The final uncertainty for $r_{\chi}$ is then the summation of four uncertainties. The result using this method is shown in Fig. 13.

The error bar for each $r_{\chi}$ is much greater than that in Fig. 7. It is shown that different methods of computing uncertainties may produce very different results.

[1] P. K. Yeung, S. Xu, and K. R. Sreenivasan, Schmidt number effects on turbulent transport with uniform mean scalar gradient, Phys. Fluids 14, 4178 (2002).

[2] P. K. Yeung, S. Xu, D. A. Donzis, and K. R. Sreenivasan, Simulations of three-dimensional turbulent mixing for Schmidt numbers of the order 1000, Flow, Turbulence Combust. 72, 333 (2004).

[3] P. A. O'gorman and D. I. Pullin, Effect of Schmidt number on the velocity-scalar cospectrum in isotropic turbulence with a mean scalar gradient, J. Fluid Mech. 532, 111 (2005).

[4] P. K. Yeung and K. R. Sreenivasan, Direct numerical simulation of turbulent mixing at very low Schmidt number with a uniform mean gradient, Phys. Fluids 26, 015107 (2014).

[5] T. Gotoh and T. Watanabe, Scalar flux in a uniform mean scalar gradient in homogeneous isotropic steady turbulence, Phys. D (Amsterdam, Neth.) 241, 141 (2012).

[6] D. A. Donzis, K. R. Sreenivasan, and P. K. Yeung, Scalar dissipation rate and dissipative anomaly in isotropic turbulence, J. Fluid Mech. 532, 199 (2005).

[7] D. A. Donzis, P. K. Yeung, and K. R. Sreenivasan, Dissipation and enstrophy in isotropic turbulence: Resolution effects and scaling in direct numerical simulations, Phys. Fluids 20, 045108 (2008).

[8] D. A. Donzis, K. R. Sreenivasan, and P. K. Yeung, The Batchelor spectrum for mixing of passive scalars in isotropic turbulence, Flow, Turbulence Combust. 85, 549 (2010).

[9] T. Watanabe and T. Gotoh, Statistics of a passive scalar in homogeneous turbulence, New J. Phys. 6, 40 (2004). 
[10] K. P. Iyer and P. K. Yeung, Structure functions and applicability of Yaglom's relation in passive-scalar turbulent mixing at low Schmidt numbers with uniform mean gradient, Phys. Fluids 26, 085107 (2014).

[11] P. L. Carroll, S. Verma, and G. Blanquart, A novel forcing technique to simulate turbulent mixing in a decaying scalar field, Phys. Fluids 25, 095102 (2013).

[12] D. Daniel, D. Livescu, and J. Ryu, Reaction analogy based forcing for incompressible scalar turbulence, Phys. Rev. Fluids 3, 094602 (2018).

[13] K. J. Rah, C. Dhandapani, and G. Blanquart, Derivation of a realistic forcing term to reproduce the turbulent characteristics of round jets on the centerline, Phys. Rev. Fluids 3, 084606 (2018).

[14] T. S. Lundgren, Linearly forced isotropic turbulence, in Annual Research Briefs (Center for Turbulence Research, Stanford, 2003), pp. 461-473.

[15] G. N. Abramovich, The Theory of Turbulent Jets (MIT Press, Cambridge, MA, 1963).

[16] S. B. Pope, Turbulent Flows (Cambridge University Press, Cambridge, UK, 2001).

[17] V. D. Zimin and P. G. Frik, Averaged temperature fields in asymmetrical turbulent streams over localized heat sources, Fluid Dyn. 12, 335 (1977).

[18] A. D. Birch, D. R. Brown, M. G. Dodson, and J. R. Thomas, The turbulent concentration field of a methane jet, J. Fluid Mech. 88, 431 (1978).

[19] P. N. Papanicolaou and E. J. List, Investigations of round vertical turbulent buoyant jets, J. Fluid Mech. 195, 341 (1988).

[20] P. C. K. Chu, J. H. Lee, and V. H. Chu, Spreading of turbulent round jet in coflow, J. Hydraul. Eng. 125, 193 (1999).

[21] D. R. Webster, P. J. W. Roberts, and L. Ra'ad, Simultaneous DPTV/PLIF measurements of a turbulent jet, Exp. Fluids 30, 65 (2001).

[22] N. R. Panchapakesan and J. L. Lumley, Turbulence measurements in axisymmetric jets of air and helium. Part 2. Helium jet, J. Fluid Mech. 246, 225 (1993).

[23] S. M. Anderson and K. Bremhorst, Investigation of the flow field of a highly heated jet of air, Int. J. Heat Fluid Flow 23, 205 (2002).

[24] A. Darisse, J. Lemay, and A. Benaïssa, Budgets of turbulent kinetic energy, Reynolds stresses, variance of temperature fluctuations and turbulent heat fluxes in a round jet, J. Fluid Mech. 774, 95 (2015).

[25] R. A. Antonia, B. R. Satyaprakash, and F. Hussain, Measurements of dissipation rate and some other characteristics of turbulent plane and circular jets, Phys. Fluids 23, 695 (1980).

[26] E. Ruffin, R. Schiestel, F. Anselmet, M. Amielh, and L. Fulachier, Investigation of characteristic scales in variable density turbulent jets using a second-order model, Phys. Fluids 6, 2785 (1994).

[27] O. Desjardins, G. Blanquart, G. Balarac, and H. Pitsch, High order conservative finite difference scheme for variable density low Mach number turbulent flows, J. Comput. Phys. 227, 7125 (2008).

[28] V. Eswaran and S. B. Pope, An examination of forcing in direct numerical simulations of turbulence, Comput. Fluids 16, 257 (1988).

[29] T. Passot and A. Pouquet, Numerical simulation of compressible homogeneous flows in the turbulent regime, J. Fluid Mech. 181, 441 (1987).

[30] P. L. Carroll, Towards understanding the mixing characteristics of turbulent buoyant flows, Ph.D. thesis, California Institute of Technology, 2014.

[31] S. Verma, Y. Xuan, and G. Blanquart, An improved bounded semi-Lagrangian scheme for the turbulent transport of passive scalars, J. Comput. Phys. 272, 1 (2014).

[32] R. Chevray and N. K. Tutu, Intermittency and preferential transport of heat in a round jet, J. Fluid Mech. 88, 133 (1978).

[33] Y. Antoine, F. Lemoine, and M. Lebouché, Turbulent transport of a passive scalar in a round jet discharging into a co-flowing stream, Eur. J. Mech. B 20, 275 (2001).

[34] D. R. Dowling and P. E. Dimotakis, Similarity of the concentration field of gas-phase turbulent jets, J. Fluid Mech. 218, 109 (1990).

[35] J. C. Duffet and A. Benaïssa, Influence of initial conditions on the evolution towards similarity of passive scalar in turbulent round jets, Exp. Therm. Fluid Sci. 44, 834 (2013).

[36] K. R. Sreenivasan, On local isotropy of passive scalars in turbulent shear flows, Proc. R. Soc. London A 434, 165 (1991). 
[37] K. R. Sreenivasan, The passive scalar spectrum and the Obukhov-Corrsin constant, Phys. Fluids 8, 189 (1996).

[38] S. K. Lee, L. Djenidi, and R. A. Antonia, Spectral method for determining mean dissipation rates of turbulent kinetic energy and passive scalar variance, in Proceedings of the 18th Australasian Fluid Mechanics Conference, Launceston, Australia (2012), Vol. 259.

[39] J. Beran, Statistics for Long-Memory Processes (CRC Press, Boca Raton, FL, 1994). 